\title{
Evaluation of La-Doped Mesoporous Bioactive Glass as Adsorbent and Photocatalyst for Removal of Methylene Blue from Aqueous Solution
}

\author{
Liying Li, ${ }^{1,2}$ Huanrui Shi, ${ }^{2}$ Lu Chen, ${ }^{2}$ Qianxuan Yuan, ${ }^{2}$ Xi Chen, ${ }^{1,3}$ and Weijian Lin ${ }^{2}$ \\ ${ }^{1}$ State Key Laboratory of Separation Membranes and Membrane Processes, Tianjin Polytechnic University, Tianjin 300387, China \\ ${ }^{2}$ School of Environmental and Chemical Engineering, Tianjin Polytechnic University, Tianjin 300387, China \\ ${ }^{3}$ School of Materials Science and Engineering, Tianjin Polytechnic University, Tianjin 300387, China \\ Correspondence should be addressed to Liying Li; liliying_tjpu@163.com
}

Received 15 September 2015; Accepted 12 October 2015

Academic Editor: Wanjun Wang

Copyright (C) 2015 Liying Li et al. This is an open access article distributed under the Creative Commons Attribution License, which permits unrestricted use, distribution, and reproduction in any medium, provided the original work is properly cited.

\begin{abstract}
A series of La-doped mesoporous bioactive glass (BG-La) materials with excellent biosafety and hypotoxicity have been prepared and tested as adsorbent. The study was aimed to evaluate the possibility of utilizing BG-La for the adsorptive removal of methylene blue (MB) from aqueous solution and test the adsorption and desorption behavior of this new material. The process parameters affecting adsorption behaviors such as $\mathrm{pH}$, contact time, and initial concentration and the photocatalytic degradation of $\mathrm{MB}$ were systematically investigated. The result showed that BG-La had excellent removal rate $(R)$ of MB, and BG-La showed better photocatalytic effect than undoped mesoporous bioactive glass (BG). Furthermore, the MB loaded BG-La was easily desorbed with acid solution due to its electronegativity and mesoporous structure. The result indicated that these materials can be employed as candidates for removal of dye pollutant owing to their high removal rate, excellent photocatalytic effect, desorption performance, and their reusability.
\end{abstract}

\section{Introduction}

Environmental pollution is mainly due to the improper waste management of industrial production process and no environmental protection consciousness of wanton development, and it is one of the concerns for the sustainable development of the earth. In particular, organic pollutants [1] that include dyes $[2,3]$ and agricultural wastes [4] are released into the environment and can be transported over long distances in the atmosphere, water, and land. Therefore, in order to purify water, many technology have been developing and developed, such as membrane filtration technology [5], coagulation and flocculation technology [6], electrochemical oxidation technology [7], adsorption technology [8-10], biological treatment technology $[6,11]$, and advanced oxidation process (AOP) [12]. However, some of the techniques shown in the effect are not ideal in practice. But because of easy operation and high cost-effectiveness, adsorption technology is considered to have good industrial application prospect of water treatment technology. Mesoporous materials, as a new adsorbent, caused wide public concern by their large specific surface area, high porosity, and so on. And mesoporous bioactive glass besides having the characteristics of traditional mesoporous materials also has high biological activity, so many researchers focus on the mesoporous bioactive glass [13]. Lanthanum has the good physical properties such as photoelectromagnetic; it can form new materials of different performance by mixing it with other materials to greatly improve their quality and performance.

But La-doped mesoporous bioactive glass materials have seldom been reported so far. In this work, La-doped mesoporous bioglass was prepared by using sol-gel method to remove methylene blue in aqueous solution, and the effect of different initial conditions on the removal efficiency was investigated. The photocatalytic activities and reusability of BG-La have also been examined. 
TABLE 1: Chemical compositions and the amounts of the reactants of different BG-La.

\begin{tabular}{|c|c|c|c|c|c|}
\hline Sample name & BG-La-1 & BG-La-2 & BG-La-3 & BG-La-4 & BG-La-5 \\
\hline Molar ratio $\mathrm{Si}: \mathrm{Ca}: \mathrm{P}: \mathrm{La}$ & $100: 0: 0: 1$ & $90: 5: 5: 1$ & $80: 15: 5: 1$ & $70: 25: 5: 1$ & $60: 35: 5: 1$ \\
\hline
\end{tabular}

\section{Materials and Methods}

2.1. Reagents and Solutions. Ethyl orthosilicate (TEOS), lanthanum nitrate $\left(\mathrm{La}\left(\mathrm{NO}_{3}\right)_{3} \cdot 6 \mathrm{H}_{2} \mathrm{O}\right)$, anhydrous ethanol, triethyl phosphate (TEP), and calcium nitrate $\left(\mathrm{Ca}\left(\mathrm{NO}_{3}\right)_{2} \cdot 4 \mathrm{H}_{2} \mathrm{O}\right)$ were purchased from Tianjin Kermel Chemical Reagent Co., Ltd. Polyoxyethylene-polyoxypropylene-polyoxyethylene three block polymer (P123) was purchased from Aldrich. Concentrated hydrochloric acid was purchased from Tianjin Wind Ship Chemical Regent Co., Ltd. Methylene blue was purchased from Tianjin New Fine Chemical Industry Development Center. The above reagents are analytically pure.

2.2. Synthesis of BG-La. A series of BG-La $\left(\mathrm{SiO}_{2}-\mathrm{CaO}-\right.$ $\left.\mathrm{P}_{2} \mathrm{O}_{5}-\mathrm{La}\right)$ solutions with five different chemical compositions were prepared following a previously reported method [14]. Briefly, $4.0 \mathrm{~g}$ of P123 were dissolved in $50 \mathrm{~mL}$ of ethanol as solution A; predetermined amount of TEOS, $\mathrm{Ca}\left(\mathrm{NO}_{3}\right)_{2} \cdot 4 \mathrm{H}_{2} \mathrm{O}$, TEP, and $1.0 \mathrm{~mL}$ of $0.5 \mathrm{M} \mathrm{HCl}$ were dissolved in $10 \mathrm{~mL}$ of ethanol and poured into solution $\mathrm{A} ; 0.187 \mathrm{~g}$ of $\mathrm{La}\left(\mathrm{NO}_{3}\right)_{3} \cdot 6 \mathrm{H}_{2} \mathrm{O}$ was dissolved in $20 \mathrm{~mL}$ of distilled water and poured into solution $\mathrm{A}$ and stirred at room temperature for $24 \mathrm{~h}$. Once the samples were completely dried, they were calcined at $800^{\circ} \mathrm{C}$ for $10 \mathrm{~h}$ in a flow of air yielding the BG-La. The amounts of reactants, final compositions, and the sample names are listed in Table 1 . The molar ratio (percentage) of $\mathrm{SiO}_{2}(\mathrm{~S})$ and $\mathrm{CaO}(\mathrm{C})$ was used to denote BG-Las with different compositions and the $\mathrm{P}_{2} \mathrm{O}_{5}$ content was kept $5 \%$ in all the samples.

2.3. Characterizations. The crystal phase and structure of the samples were identified by an X-ray powder diffractometer (XRD, D8-Advance, Bruker, Germany) using Cu $\mathrm{K} \alpha$ Radiation $(\lambda=1.54178 \AA)$ and a fixed power source $(40.0 \mathrm{kV}, 40.0 \mathrm{~mA})$. The X-ray photoelectron spectra (XPS) were carried out on ESCALAB 250 electron energy spectrometer (Thermo Fisher Scientific, USA) using Monochromated Al Kalph $150 \mathrm{~W}$ as the X-ray excitation source. The morphology and microstructure of the samples were examined by field emission scanning electron microscopy (SEM, Hitachi X-650B, Japan) and high resolution transmission electron microscopy (TEM, JEM-2010, JEOL, Japan). $\mathrm{N}_{2}$ adsorption-desorption isotherms were obtained on a Chemisorption-Physisorption Analyzer (Autosorb-1-C, Quantachrome, USA) at $77 \mathrm{~K}$ under continuous adsorption condition. BET and $\mathrm{BJH}$ analyses were used to determine the surface area, the pore size distribution, and the pore volume.

2.4. Batch Adsorption Experiments. Adsorption experiments were carried out by contacting $0.60 \mathrm{~g}$ of BG-La with $25 \mathrm{~mL}$ of $\mathrm{MB}$ solution of different initial concentrations $(5-50 \mathrm{mg} / \mathrm{L})$. A series of such conical flasks were then shaken at a constant speed of $150 \mathrm{rpm}$ in a shaking water bath in $298 \mathrm{~K}$. The solution was then centrifuged at $5000 \mathrm{rpm}$ for $5 \mathrm{~min}$ and the initial $\left(C_{0}\right)$ and equilibrium $(C)$ concentrations were analyzed using a UV spectrophotometer (Beijing Analysis of General Instrument Co., Ltd., TU-1810, China) by monitoring the absorbance changes at a wavelength of maximum absorbance $(668 \mathrm{~nm})$. The MB removal efficiency $(R)$ was evaluated by the following formula:

$$
R=\frac{100\left(C_{0}-C\right)}{C_{0}}
$$

where $C_{0}$ and $C$ are, respectively, the initial and the final concentrations $(\mathrm{mg} / \mathrm{L})$. Experiments were conducted at various time intervals to determine the kinetic parameters.

To study the effect of $\mathrm{pH}$ on adsorption capacity, the adsorption experiments were performed at various $\mathrm{pH}$ values. The $\mathrm{pH}$ of solution was controlled to 2.0-12.0 after the sorption equilibrium by adding $\mathrm{HCl}$ or $\mathrm{NaOH}$ solution.

2.5. Photocatalysis. The photocatalytic activity was evaluated by measuring the decomposition of $\mathrm{MB}$ solutions irradiated under ultraviolet light (UV lamp, Philips, TUV 4 W/G4T5, at $365 \mathrm{~nm}$ ). A custom-built well-stirred tank photoreactor, consisting of six $4 \mathrm{~W}$ quartz tube (i.d. $1 \mathrm{~cm}$ ) arranged to receive equal light intensity was utilized in the tests. A high stirring speed of $400 \mathrm{rpm}$ was used to avoid external mass transfer limitations during the photocatalytic reaction. The photocatalyst was settled in the aqueous suspension for 120 min in darkness to reach adsorption equilibrium with $\mathrm{MB}$ before the experiment. During the experiment, $3.0 \mathrm{~mL}$ of samples was taken at various time intervals and then centrifuged at $5000 \mathrm{rpm}$ for $5 \mathrm{~min}$ and the clear supernatant was analyzed using UV spectrophotometer as discussed later.

\section{Results and Discussion}

3.1. Characterization of BG-La. Typical XRD pattern of BGLa-2 in the small-angle region is shown in Figure 1(a). Smallangle XRD patterns exhibit diffraction peaks in the range of $0.5-1.5^{\circ}$; the three obvious diffraction peaks, respectively, belonged to the P6 mm crystal system SBA-15 (100), (110), and (200) crystal plane, which proves the existence of the characteristics of the six-party symmetry structure of BGLa-2 materials [15]. Furthermore, it can be observed from Figure 1(b) that there are no diffraction peaks of lanthanum (III) appearing at $2 \theta=10-35^{\circ}$, which suggests that lanthanum (III) little doped amount or lanthanum (III) were scattered in the biological glass.

The TEM analysis was performed to investigate the microstructure and porous arrangement mode of the BGLa materials. Results are shown in Figures 2(a)-2(e). BGLa materials had uniform and regularly arrayed pores with average pore size. And as the red marks shown in Figures 


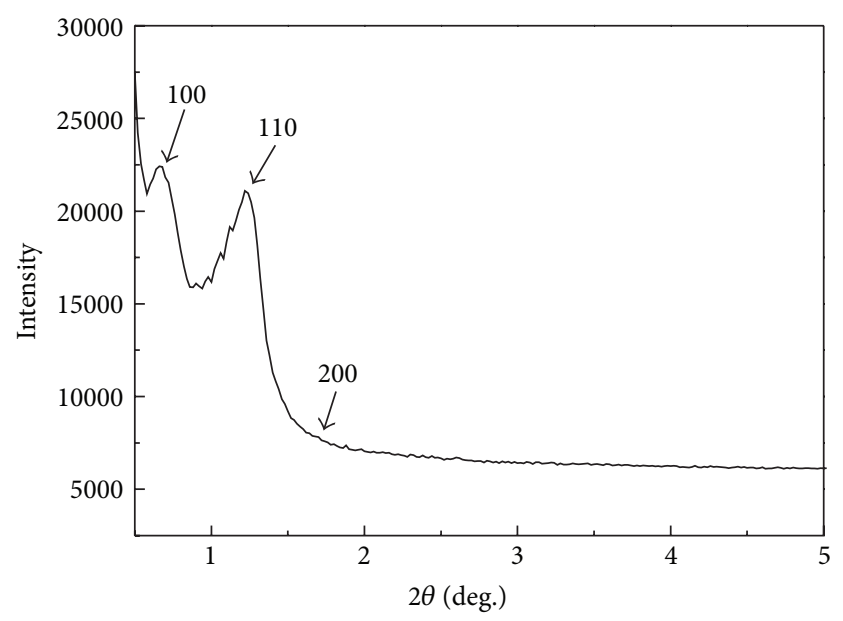

(a)

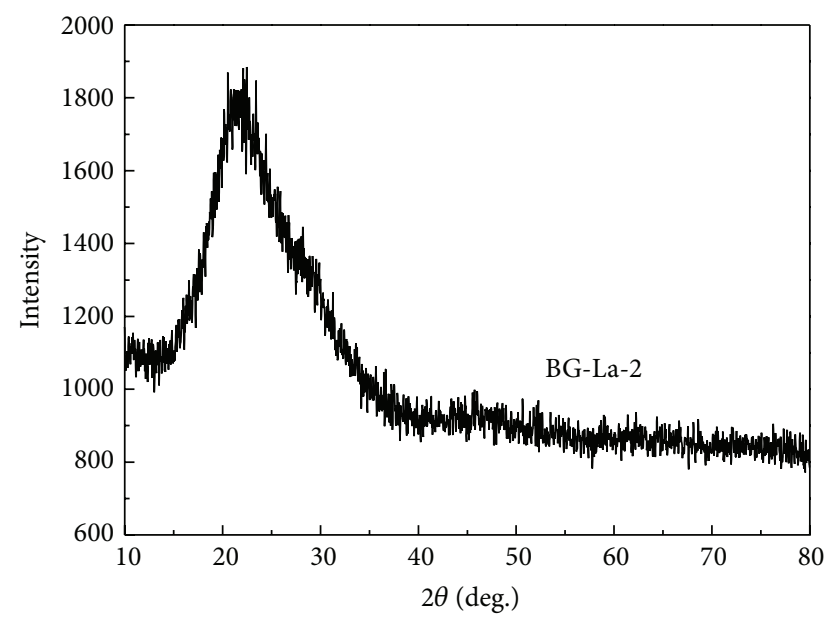

(b)

FIGURE 1: (a) Small-angle XRD pattern of BG-La-2; (b) XRD pattern of BG-La-2.

TABLE 2: Textural and porous parameters of BG-La materials.

\begin{tabular}{lccc}
\hline Adsorbent & $\begin{array}{c}\text { Surface area } \\
\left(\mathrm{m}^{2} \mathrm{~g}^{-1}\right)\end{array}$ & $\begin{array}{c}\text { Pore volume } \\
\left(\mathrm{ccg}^{-1}\right)\end{array}$ & $\begin{array}{c}\text { Pore diameter } \\
(\mathrm{nm})\end{array}$ \\
\hline BG-La-1 & 293 & 0.41 & 2.6 \\
BG-La-2 & 289 & 0.30 & 2.4 \\
BG-La-3 & 303 & 0.45 & 3.1 \\
BG-La-4 & 316 & 0.49 & 3.7 \\
BG-La-5 & 311 & 0.47 & 3.5 \\
\hline
\end{tabular}

2(a), 2(b), 2(c), and 2(e), samples 1-3 and 5 have ordered twodimensional six-party channel structure, consistent with the XRD test results. The morphological structure of BG-La was characterized by SEM and shown in Figure 2(f). It could be observed that no obvious crystal was formed and the BG-La existed in amorphous state.

The $\mathrm{N}_{2}$ adsorption-desorption isotherm and the pore size distribution of BG-La-2 material are shown in Figure 3. The nitrogen adsorption-desorption isotherm can be identified as type IV isotherm characteristic of mesoporous structure. The mesoporous sample exhibits hysteresis loop at the $P / P_{0}$ range of $0.4-0.8$. The hysteresis loop is probably due to the existence of textural interparticle mesoporosity. The sample shows a pore size distribution centered between 2 and $3 \mathrm{~nm}$. The detailed textural and porous parameters are listed in Table 2.

XPS spectrums of BG-La-2 are shown in Figure 4. Five remarkable intense peaks were displayed in Figure 4(a) showing the main elements in samples of BG-La-2. The peak at $854.8 \mathrm{eV}$ correspond to La3d $\mathrm{d}_{3 / 2}$ XPS spectrum (Figure 4(b)). And the two peaks at $841.2 \mathrm{eV}$ and $836.4 \mathrm{eV}$ both correspond to La3 $_{5 / 2}$ (Figure 4(b)); this is bimodal phenomenon; the emergence and intensity of the bimodal phenomenon reflect the ability of lanthanum to get $\mathrm{O} 2 \mathrm{p}$ electronic ability, namely, the size of the La-O covalent [16]. The above results suggest the presence of $\mathrm{La}_{2} \mathrm{O}_{3}$ in BG-La materials [17].

\subsection{Effects of Operating Conditions on $M B$ Adsorption}

3.2.1. Effect of Contact Time. Under the same initial conditions BG-La-1 5, respectively, tested the adsorption equilibrium time and the results were shown in Figure 5. BG-La-1 and 2 reached adsorption equilibrium within $2 \mathrm{~h}$ as shown in Figure 5, and BG-La-3 5 reached adsorption equilibrium within $6 \mathrm{~h}$. The removal rate $R(\%)$ of BG-La-1 and 2 had reached more than $95 \%$ within $5 \mathrm{~min}$, and after reaching adsorption equilibrium the removal rate was close to $100 \%$; this also reflected their good mesoporous structure.

3.2.2. Effect of Initial Solution $p H$. The solution $\mathrm{pH}$ is another important factor in determining the adsorption properties of adsorbent. Figure 6 displays the variation in the amount of adsorbed MB by BG-La adsorbent with the initial solution $\mathrm{pH}$ ranging from 2 to 12 . It is noted that adsorption of $\mathrm{MB}$ onto BG-La-2 is drastically influenced by initial $\mathrm{pH}$ and the removal rate increased with the increase of $\mathrm{pH}$ value. The maximum dye adsorption was observed at $\mathrm{pH}$ 10.0. Analyzed from the structure of adsorbent and contaminate molecule, it is known that the adsorption of these charged dye groups onto the adsorbent surface is primarily influenced by the surface charge which is in turn influenced by the solution $\mathrm{pH}$ [18].

3.2.3. Effect of Initial MB Concentrations. Figure 7 shows the effects of initial MB concentrations $(5,10,20,30,40$, and $50 \mathrm{mg} / \mathrm{L}$ ) on BG-La-1 5 and undoped mesoporous bioactive glasses (BG-1 5). It can be easily observed that the removal rates of BG-La-1 5 are higher than BG-1 5, and the removal rate decreased with the increase of initial $\mathrm{MB}$ concentrations. As shown in Figure 7(b), BG-La-2 keeps a high removal efficiency at different initial concentrations, and its removal rate is above $99 \%$.

3.3. Adsorption Kinetics. In our earlier study, it is noticed that the adsorption capacities of BG-La have direct correlation with its surface area and pore structure. Thus, in this section, 

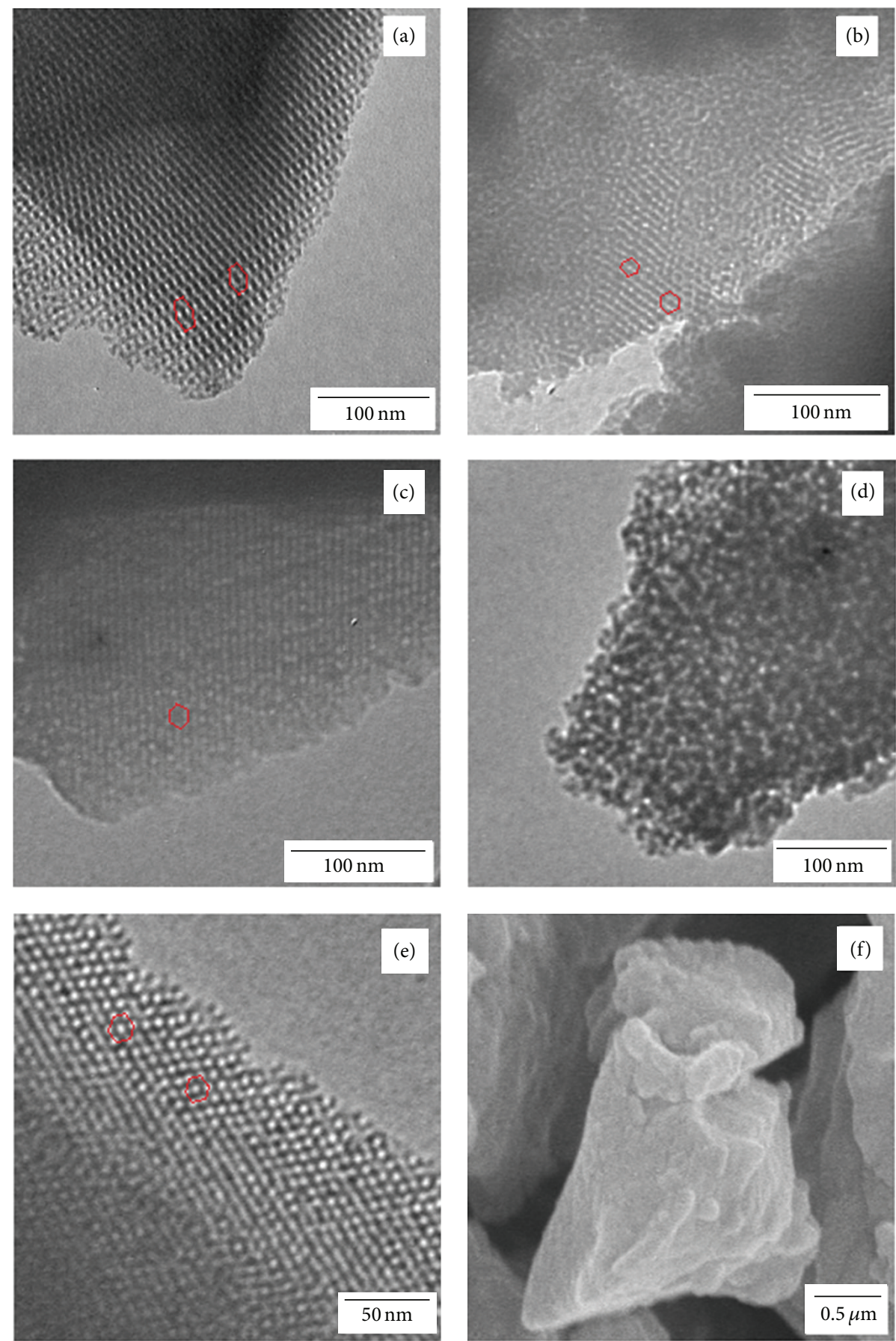

Figure 2: (a) TEM image of BG-La-1, (b) TEM image of BG-La-2, (c) TEM image of BG-La-3, (d) TEM image of BG-La-4, (e) TEM image of BG-La-5, and (f) SEM image of BG-La-2.

BG-La-2 which have the largest surface area and regular porous structure were tested as optimized adsorbents.

A study of adsorption kinetics is expected as it can provide information of adsorption mechanism. The pseudofirst-order kinetic model and pseudo-second-order kinetic model were employed to investigate the kinetics of adsorption of MB onto BG-La. The pseudo-first-order kinetic model is expressed by the following equation:

$$
\ln \left(q_{e}-q_{t}\right)=\ln q_{e}-k_{1} t
$$

where $k_{1}$ is the rate constant of pseudo-first-order reaction $\left(\min ^{-1}\right), q_{e}$ is the removal amount of $\mathrm{MB}$ at equilibrium 


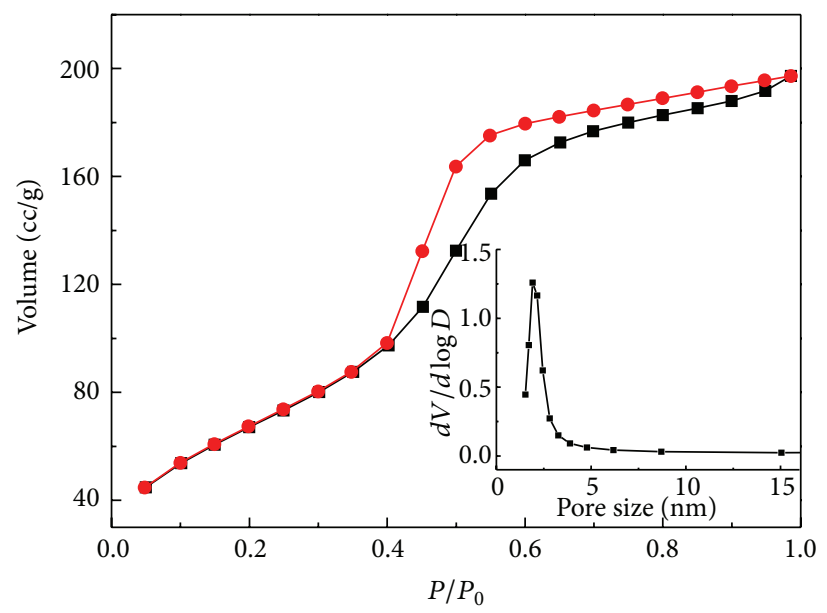

FIGURE 3: Nitrogen adsorption-desorption isotherm and pore size distribution curves of BG-La-2.

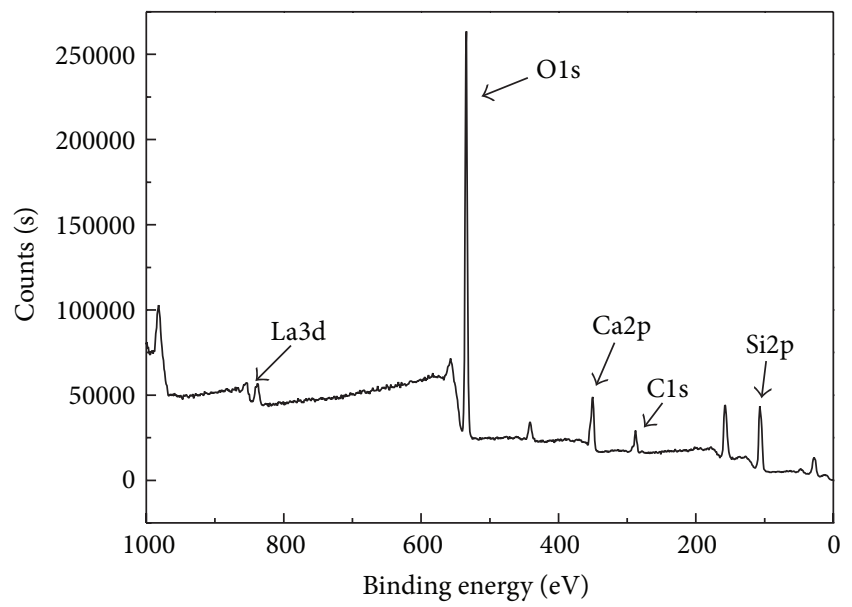

(a)

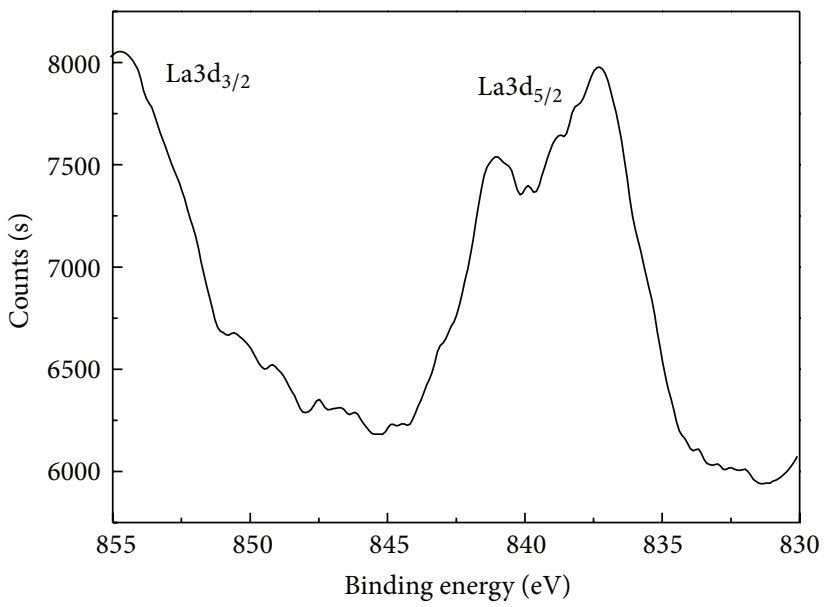

(b)

FIGURE 4: (a) The main elements XPS spectrum of BG-La-2; (b) lanthanum XPS spectrum of BG-La-2.

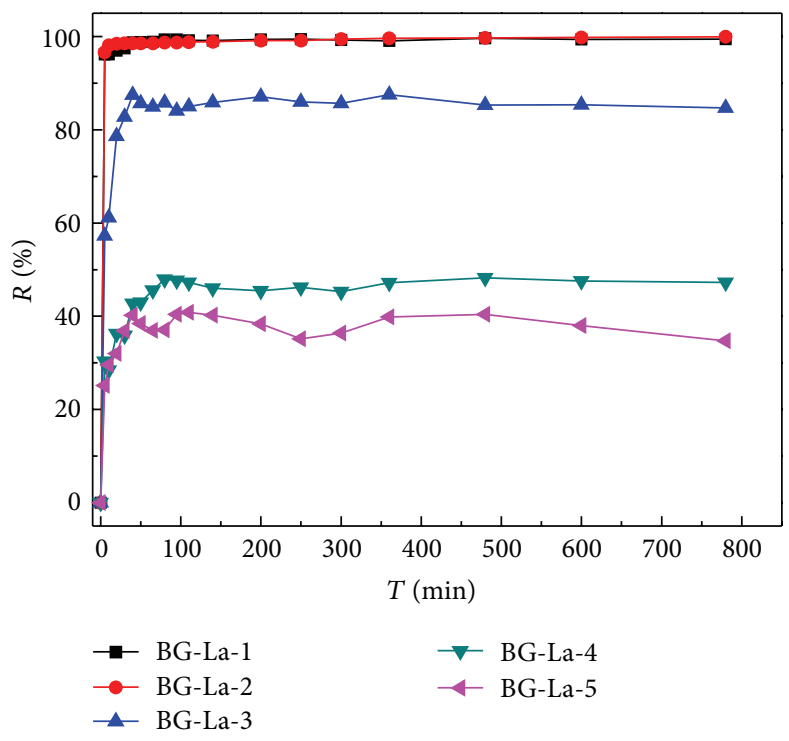

FIGURE 5: Effect of contact time on MB adsorption. 
TABLE 3: Comparison of kinetics parameters of MB onto BG-La.

\begin{tabular}{lcccccc}
\hline \multirow{2}{*}{ Adsorbent } & \multirow{2}{*}{$q_{e, \exp }$} & \multicolumn{2}{c}{ Pseudo-first-order kinetic model } & \multicolumn{2}{c}{ Pseudo-second-order kinetic model } \\
& & $q_{1 e, \text { cal }}(\mathrm{mg} / \mathrm{g})$ & $k_{1}\left(\mathrm{~min}^{-1}\right)$ & $R^{2}$ & $q_{2 e, \text { cal }}(\mathrm{mg} / \mathrm{g})$ & $k_{2}(\mathrm{~g} /(\mathrm{mg} \mathrm{min}))$ \\
\hline BG-La-1 & 8.64 & 0.22 & 0.012 & 0.8636 & 8.62 & 0.337 \\
BG-La-2 & 8.66 & 0.14 & 0.003 & 0.9021 & 8.66 & 0.136 \\
BG-La-3 & 7.59 & 1.55 & 0.014 & 0.8175 & 7.40 & 0.9999 \\
BG-La-4 & 4.18 & 1.37 & 0.018 & 0.9152 & 4.14 & 0.363 \\
BG-La-5 & 3.54 & 0.94 & 0.015 & 0.9192 & 3.18 & 0.047 \\
\hline
\end{tabular}

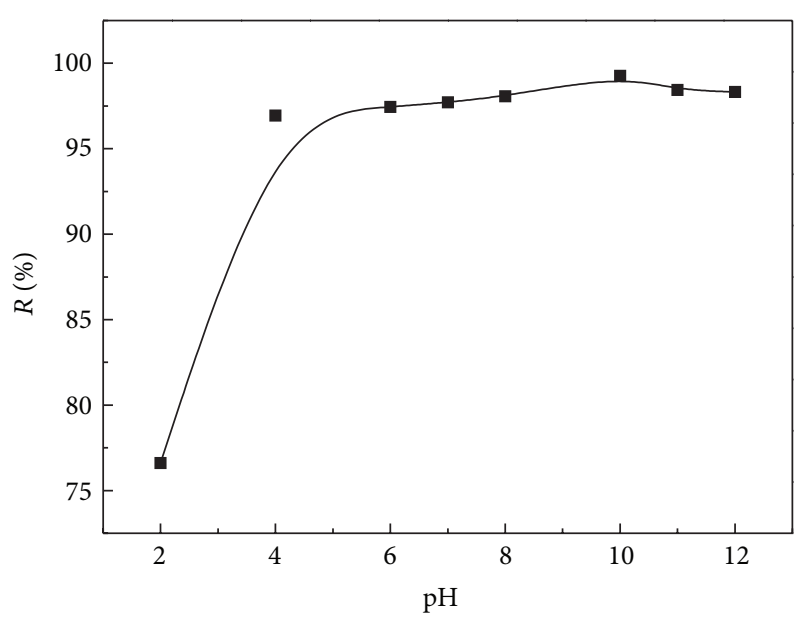

FIGURE 6: Influence of solution initial pH of BG-La-2.

(mg/g) and $q_{t}$ is the removal amount of $\mathrm{MB}$ at any time $t$ (min). The adsorption kinetics is shown in Figure 8 and the values of $k_{1}$ and $q_{e}$ are presented in Table 3 . The values of experimental $q_{e}\left(q_{e, \exp }\right)$ do not agree with the calculated $q_{e}\left(q_{e, \text { cal }}\right)$ and the values of correlation coefficient $\left(R^{2}\right)$ are relatively low. This indicated that the adsorption process did not fit to the pseudo-first-order model.

The pseudo-second-order model is expressed as

$$
\frac{t}{q_{t}}=\frac{1}{k_{2} q_{e}^{2}}+\frac{t}{q_{e}},
$$

where $k_{2}$ is the rate constant of pseudo-second-order reaction $(\mathrm{g} /(\mathrm{mg} \mathrm{min}))$, which can be calculated by the slope of plot $t / q_{t}$ versus $t$. The values of $R^{2}$ were greater than 0.99 for all MB concentrations. It can be seen from Figure 9 and Table 3 that a good agreement was involved between the experimental $q_{e}\left(q_{e, \text { exp }}\right)$ and the calculated $q_{e}\left(q_{e, \text { cal }}\right)$ values, indicating the applicability of the pseudo-second-order to describe the adsorption process of dye MB on BG-La.

The kinetic data were analyzed by the aforementioned kinetic equations, and corresponding parameters were calculated in Table 3 . The values of correlation coefficient $\left(R^{2}\right)$ obtained from pseudo-second-order kinetics were higher $(>0.99)$ than those from pseudo-first-order kinetics and the values of $q_{2 e \text {,cal }}$ were closer to experimentally obtained adsorption capacity $\left(q_{e, \exp }\right)$, and thus pseudo-second-order kinetics could reasonably describe the adsorption process for the adsorption systems.
3.4. Adsorption Isotherms. Adsorption isotherm is important to describe how solutes interact with adsorbents, and it is critical in optimizing an adsorption process to indicate how the adsorbed molecules distribute themselves between liquid and solid phases until the adsorption process reaches an equilibrium state. The different isotherm models are used to analyze adsorption equilibrium data. An accurate mathematical depiction for adsorption process is indispensable for reliable prediction for adsorption process and quantitative comparison of adsorption behaviors. In the present study, the adsorption equilibrium data were analyzed by the Langmuir and Freundlich isotherm models.

The Langmuir isotherm model is applied to analyze the interaction between the MB molecule and BG-La adsorbent when the adsorption process reaches equilibrium. It is assumed that the adsorption process is monolayer, which means no further adsorption occurs once adsorption takes place at specific sites on the adsorbent. Hence, the adsorption is strongly related to the surface area of the adsorbent and the driving force such as London-van der Waals force. The relationship is expressed as follows:

$$
\frac{c_{e}}{Q_{e}}=\frac{c_{e}}{Q_{m}}+K_{L} Q_{m}
$$

where $c_{e}$ is the liquid-phase concentration of adsorbate at equilibrium (mg/L), $Q_{e}$ is the amount of adsorbate adsorbed at equilibrium $(\mathrm{mg} / \mathrm{g}), Q_{m}$ is the maximum adsorption capacity $(\mathrm{mg} / \mathrm{g})$, and $K_{L}$ is the Langmuir constant related to the energy of adsorption $(\mathrm{L} / \mathrm{mg})$.

The Freundlich isotherm model is an empirical equation and was generally applied to multilayer adsorption, with nonuniform distribution of adsorption heat and affinities over the heterogeneous surface of absorbent. The equation is described as follows:

$$
Q_{e}=K_{F} C_{e}^{1 / n}
$$

where $K_{F}$ and $n$ are the Freundlich constants which represent the adsorption capacity and adsorption strength, respectively. The magnitude of $1 / n$ qualifies the degree of heterogeneity of the adsorbent surface and the favorability of adsorption. If $n>1$, the adsorption process is favorable and new adsorption sites form on the surface of the adsorbent. $K_{F}$ and $n$ can be obtained from the intercept and slope of the linear plot of $\ln$ $q_{e}$ versus $\ln c_{e}$.

The relative parameters based on Langmuir and Freundlich were calculated and listed in Table 4, and the Freundlich isotherms of MB on BG-La were shown in Figure 10. 

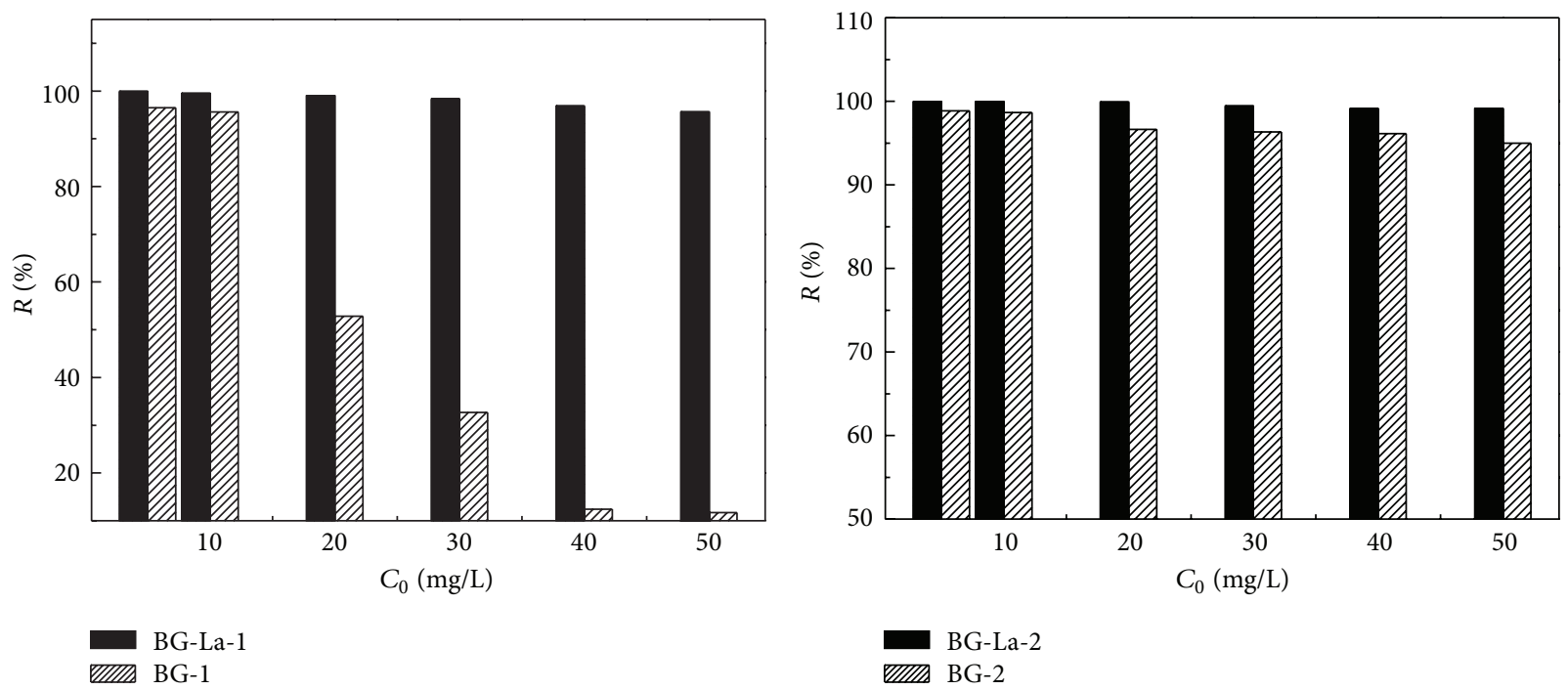

(a)

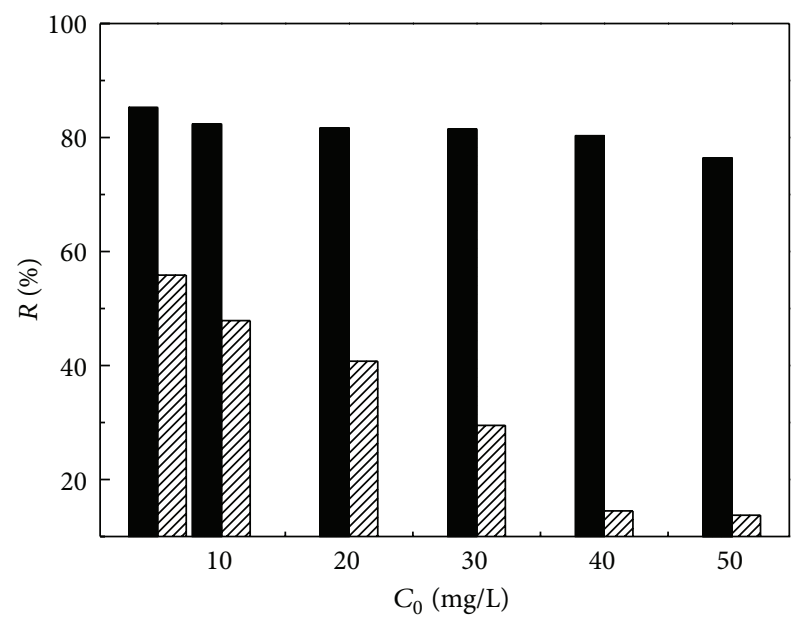

(b)

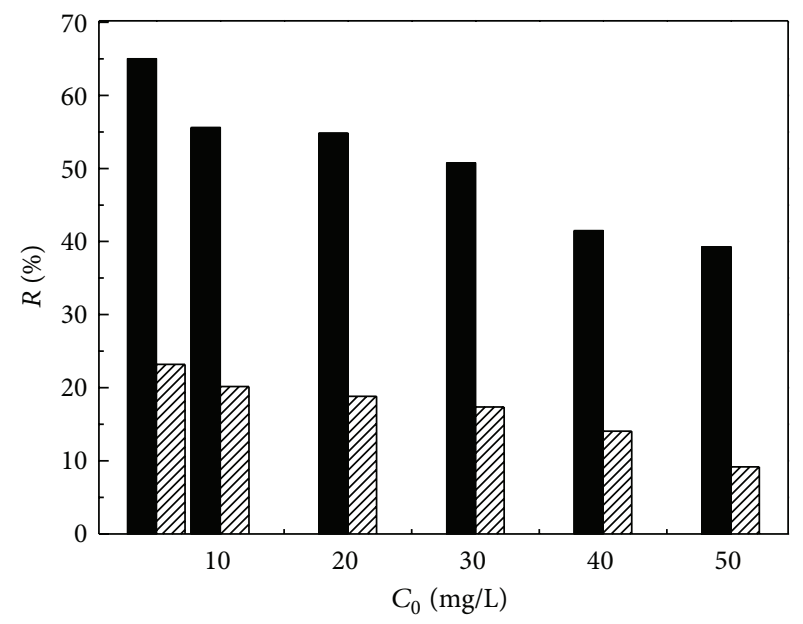

BG-La-3 पाm BG-3

BG-La-4

एखा BG-4

(c)

(d)

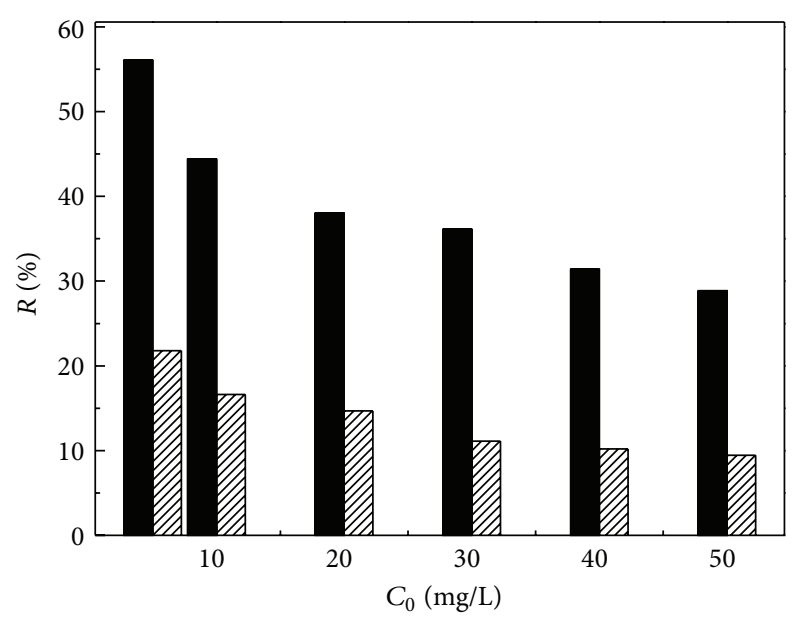

BG-La-5

प्याग BG-5

(e)

FIGURE 7: Effect of initial MB concentrations on BG-La-1 5 and BG-1 5. 


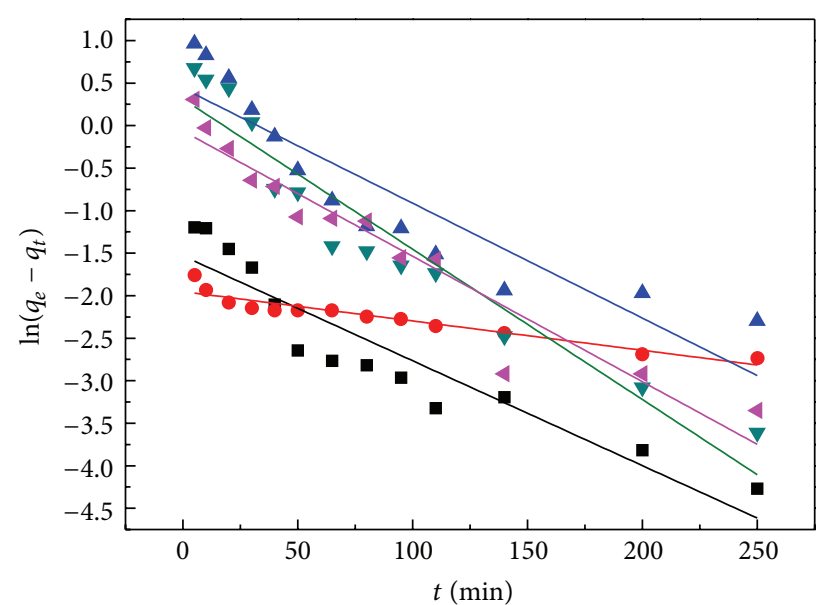

- BG-La-1

- BG-La-2

A BG-La-3

FIgURE 8: The pseudo-first-order kinetics of MB by BG-La.

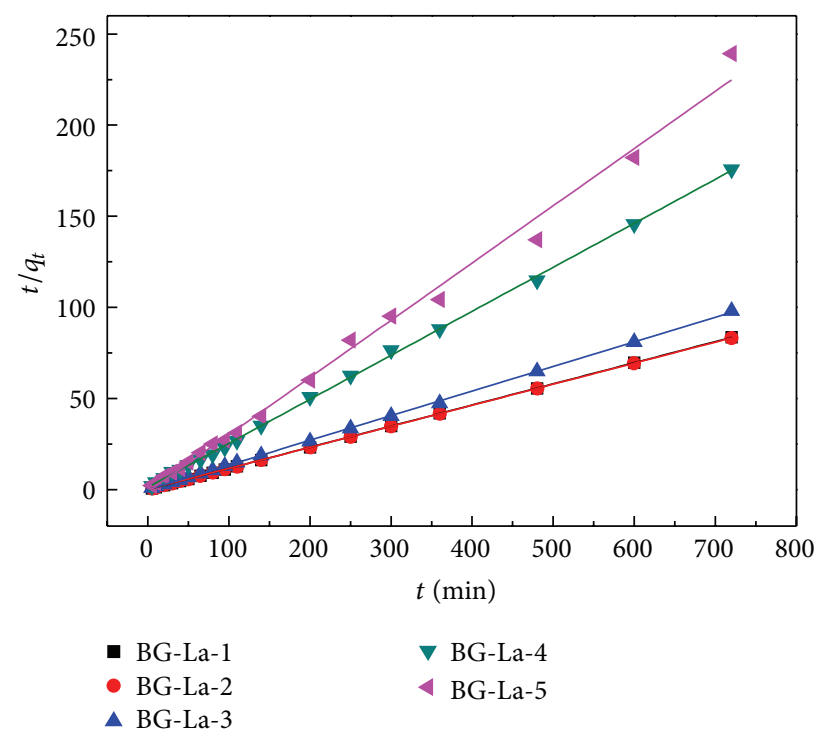

FIGURE 9: The pseudo-second-order kinetics of MB by BG-La.

BG-La-3 5 do not conform to the Langmuir isotherm model. According to coefficient $\left(R^{2}\right)$, it is suggested that the adsorption of MB on the BG-La is better modeled by the Freundlich isotherm than the Langmuir isotherm. These results indicate that the MB molecules are not bonded as a monolayer on the surface of the BG-La adsorbent. The higher adsorption capacity of $\left(Q_{m}\right)$ of BG-La-2 may be attributed to the larger surface areas and mesoporous structure.

3.5. Thermodynamic Parameters. Thermodynamics parameters can estimate the effect of temperature on $\mathrm{MB}$ adsorption onto BG-La and reflect the adsorption mechanism and behavior. It includes enthalpy change $\left(\Delta H^{0}\right)$, Gibbs free

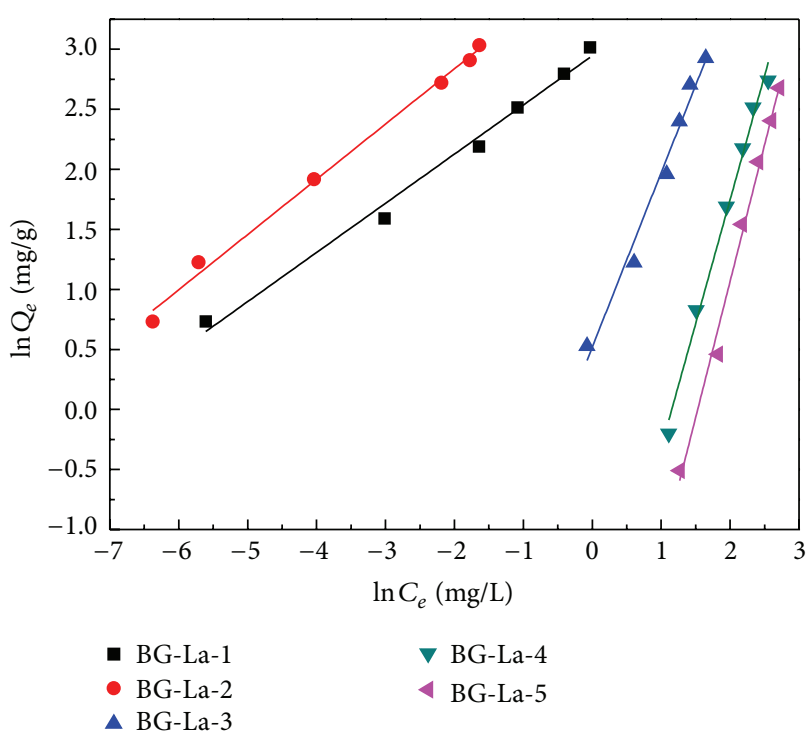

FIgURE 10: The Freundlich isotherm of MB on BG-La.

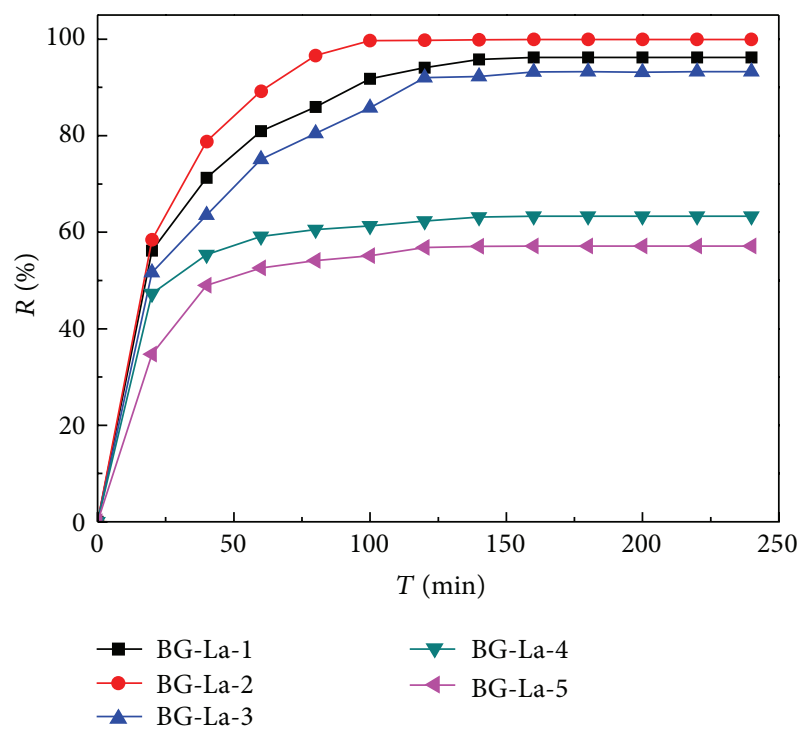

FIGURE 11: $R$ (\%) plotted against time for BG-La materials under UV light irradiation.

energy change $\left(\Delta G^{0}\right)$, and entropy change $\left(\Delta S^{0}\right)$, and they were evaluated by the following equations:

$$
\begin{aligned}
\Delta G^{0} & =-R T \ln \left(K_{L}\right), \\
\ln \left(K_{L}\right) & =\frac{\Delta S^{0}}{R}-\frac{\Delta H^{0}}{R T},
\end{aligned}
$$

where $K_{L}$ is the Langmuir equilibrium constant $(\mathrm{L} / \mathrm{mol}) ; R$ and $T$ represent the universal gas constant $(8.314 \mathrm{~J} / \mathrm{mol} \mathrm{K})$ and the system temperature (K). And the thermodynamic parameters are showed in Table 5.

The negative reflect of $\Delta G^{0}$ is spontaneous and feasible. In addition, the decrease in $\Delta G^{0}$ with an increase in temperature 
TABLE 4: Parameters calculated from isotherm equations for MB onto BG-La.

\begin{tabular}{lcccccc}
\hline Adsorbent & \multicolumn{3}{c}{ Langmuir isotherm } & \multicolumn{3}{c}{ Freundlich isotherm } \\
& $Q_{m}(\mathrm{mg} / \mathrm{g})$ & $K_{L}(\mathrm{~L} / \mathrm{mg})$ & $R^{2}$ & $K_{F}(\mathrm{mg} / \mathrm{g})$ & $1 / n$ & $R^{2}$ \\
\hline BG-La-1 & 20.69 & 13.73 & 0.9795 & 19.02 & 0.41 & 0.9878 \\
BG-La-2 & 16.75 & 8.29 & 0.9887 & 42.85 & 0.46 & 0.9943 \\
BG-La-3 & $\backslash$ & $\backslash$ & $\backslash$ & 1.68 & 1.45 & 0.9765 \\
BG-La-4 & $\backslash$ & $\backslash$ & $\backslash$ & 0.09 & 2.06 & 0.9891 \\
BG-La-5 & $\backslash$ & $\backslash$ & & 0.03 & 2.27 & 0.9899 \\
\hline
\end{tabular}
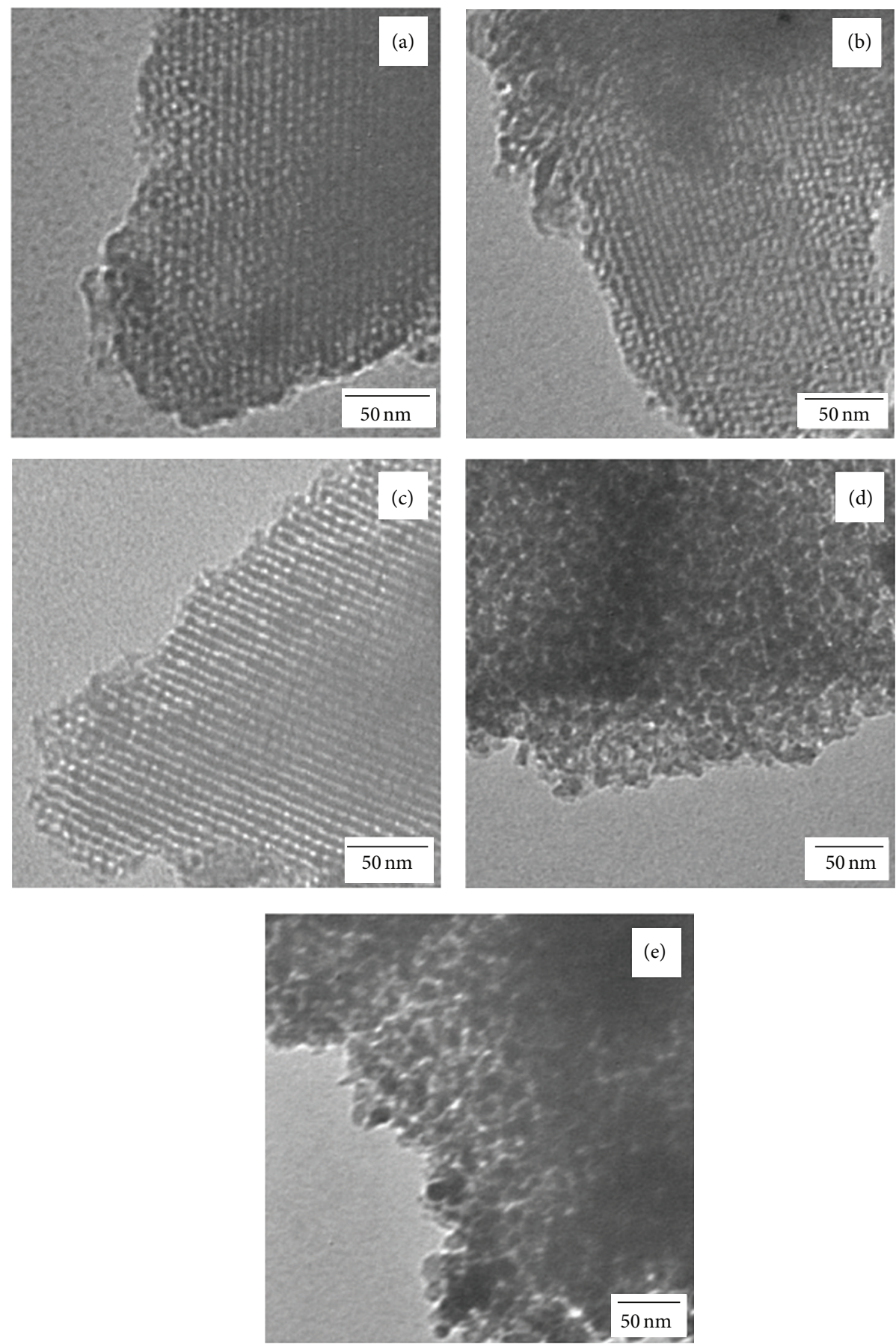

FIGURE 12: TEM images of regeneration products ((a) BG-La-1, (b) BG-La-2, (c) BG-La-3, (d) BG-La-1, and (e) BG-La-5). 
TABLE 5: Thermodynamic parameters for adsorption of MB onto BG-La-2.

\begin{tabular}{lccc}
\hline$T(\mathrm{~K})$ & $\Delta G^{0}(\mathrm{~kJ} / \mathrm{mol})$ & $\Delta H^{0}(\mathrm{~kJ} / \mathrm{mol})$ & $\Delta S^{0}(\mathrm{~J} / \mathrm{mol} \mathrm{K})$ \\
\hline 303 & -5.3 & & \\
313 & -7.9 & 73.9 & 261.5 \\
323 & -10.6 & & \\
\hline
\end{tabular}

and $\Delta H^{0}>0$ indicates that the adsorption is better at higher temperature. Furthermore, $\Delta S^{0}>0$ suggest the increasing randomness at the solid-solution interface during fixation of $\mathrm{MB}$ onto the active sites of BG-La [19].

3.6. Photocatalytic Degradation of Methylene Blue in Water. Figure 11 depicts a plot of $R(\%)$ versus time for the degradation of MB over prepared BG-La-1 5 under UV light irradiation. It is noted that the degradation of $\mathrm{MB}$ onto BGLa-1 5 is drastically influenced by UV light irradiation and the removal rate increased with the increase of time. All the materials reached degradation equilibrium within $2 \mathrm{~h}$ and BG-La-2 still maintained a high removal rate (>99\%). Furthermore, the removal rate of the degradation equilibrium of BG-La- 4 and 5 significantly increased by about $10 \%$ compared to the removal rate of the adsorption equilibrium. This suggests that the lanthanum doped mesoporous bioglass has the ability of photocatalytic degradation.

3.7. The Stability and Reusability of BG-La. The stability and reusability ability is significant for the practical application of adsorbents. Such adsorbents which have excellent adsorption capacity as well as high reusability will reduce secondary pollution and overall cost. Thus, the reuse experiments of BG-La were performed to evaluate the recyclable availability. The same mass of BG-La-1 5 was dissolved, respectively, in the same volume of $\mathrm{MB}$ solution $(20 \mathrm{mg} / \mathrm{L})$; after reaching adsorption saturation reuse experiment was carried out, the regeneration products were roasted at $500^{\circ} \mathrm{C}$ in $1 \mathrm{~h}$. Figure 12 shows the TEM images of regeneration products. The microstructure and porous arrangement mode of regeneration products were the same as the original products mostly. The adsorption-regeneration experiments were repeated for 10 times and then the datas of reusability were gotten, as shown in Figure 13. It could be observed that the decline trend of removal rate of BG-La regeneration products became slowing down after 5 times of adsorptionregeneration experiments, and BG-La-1 and 2 still kept a high removal rate $(>90 \%)$ after 10 times of adsorptionregeneration experiments. Therefore, the BG-La materials are expected to be employed repeatedly in dying wastewater treatment.

\section{Conclusions}

A facile synthesis method for BG-La was proposed in the present work; the average pore diameter is about $2.5 \mathrm{~nm}$ with the ordered two-dimensional six-party channel structure. In bath adsorption experiments, it was demonstrated that

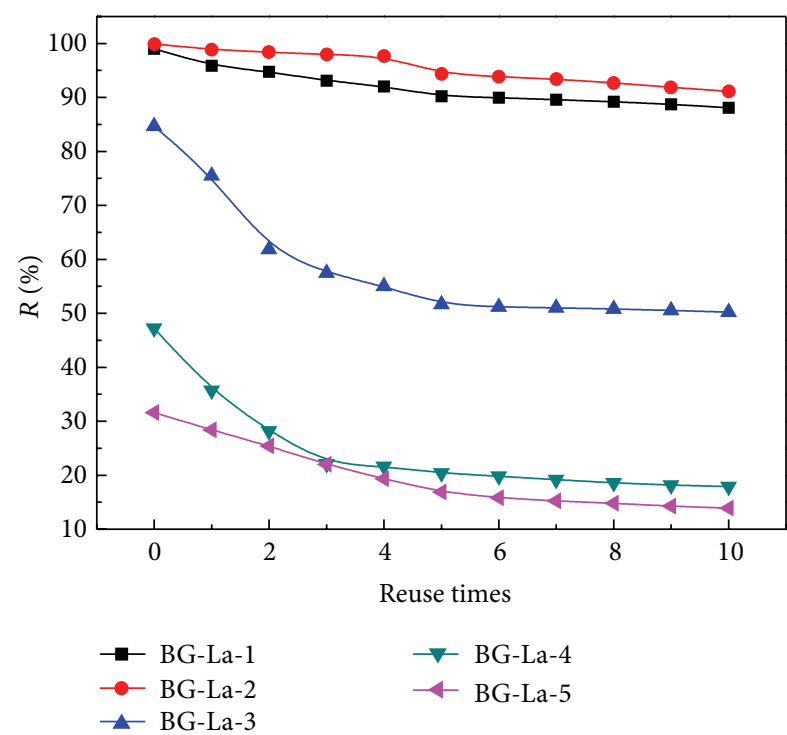

FIGURE 13: $R$ (\%) plotted against reuse times for BG-La materials.

the adsorption was tremendously affected by $\mathrm{pH}$, initial concentration, and temperature. The kinetics studies proved that the adsorption process agreed with the pseudo-secondorder kinetics. The equilibrium study was well fitted by the Freundlich isotherm model. The thermodynamics parameters indicated that the adsorption is better at higher temperature. Furthermore, the BG-La showed excellent reusability ability and photocatalytic activity which made these materials possible in environmental application.

\section{Conflict of Interests}

The authors declare that there is no conflict of interests regarding the publication of this paper.

\section{Acknowledgments}

The authors are grateful to the financial support from the National Natural Science Foundation of China (no. 30901221) and Science and Technology Commission Foundation of Tianjin (no. 10JCZDJC17100 and no. 14JCZDJC38100).

\section{References}

[1] H. Lachheb, E. Puzenat, A. Houas et al., "Photocatalytic degradation of various types of dyes (Alizarin S, Crocein Orange G, Methyl Red, Congo Red, Methylene Blue) in water by UVirradiated titania," Applied Catalysis B: Environmental, vol. 39, no. 1, pp. 75-90, 2002.

[2] A. K. Aboul-Gheit, S. M. Abdel-Hamid, S. A. Mahmoud et al., "Mesoporous Ti-MCM-41 materials as photodegradation catalysts of 2,4,6-trichlorophenol in water," Journal of Materials Science, vol. 46, no. 10, pp. 3319-3329, 2011.

[3] C. Guillard, J. Disdier, C. Monnet et al., "Solar efficiency of a new deposited titania photocatalyst: chlorophenol, pesticide and dye removal applications," Applied Catalysis B: Environmental, vol. 46, no. 2, pp. 319-332, 2003. 
[4] D. Sud and P. Kaur, "Heterogeneous photocatalytic degradation of selected organophosphate pesticides: a review," Critical Reviews in Environmental Science and Technology, vol. 42, no. 22, pp. 2365-2407, 2012.

[5] Y. Xu, R. E. Lebrun, P.-J. Gallo, and P. Blond, "Treatment of textile dye plant effluent by nanofiltration membrane," Separation Science and Technology, vol. 34, no. 13, pp. 2501-2519, 1999.

[6] S. W. Won, S. B. Choi, B. W. Chung, D. Park, J. M. Park, and Y.-S. Yun, "Biosorptive decolorization of reactive orange 16 using the waste biomass of Corynebacterium glutamicum," Industrial and Engineering Chemistry Research, vol. 43, no. 24, pp. 7865-7869, 2004.

[7] R. Pelegrini, P. Peralta-Zamora, A. R. de Andrade, J. Reyes, and N. Durán, "Electrochemically assisted photocatalytic degradation of reactive dyes," Applied Catalysis B: Environmental, vol. 22, no. 2, pp. 83-90, 1999.

[8] S. Chatterjee, S. Chatterjee, B. P. Chatterjee, A. R. Das, and A. K. Guha, "Adsorption of a model anionic dye, eosin Y, from aqueous solution by chitosan hydrobeads," Journal of Colloid and Interface Science, vol. 288, no. 1, pp. 30-35, 2005.

[9] Y. L. Dong, B. Lu, S. Y. Zang, J. Zhao, X. Wang, and Q. Cai, "Removal of methylene blue from coloured effluents by adsorption onto SBA-15," Journal of Chemical Technology and Biotechnology, vol. 86, no. 4, pp. 616-619, 2011.

[10] P. V. Messina and P. C. Schulz, "Adsorption of reactive dyes on titania-silica mesoporous materials," Journal of Colloid and Interface Science, vol. 299, no. 1, pp. 305-320, 2006.

[11] R. Ahmad, P. K. Mondal, and S. Q. Usmani, "Hybrid UASFBaerobic bioreactor for biodegradation of acid yellow-36 in wastewater," Bioresource Technology, vol. 101, no. 10, pp. 37873790, 2010.

[12] W. Guo, X. Liu, P. Huo et al., "Hydrothermal synthesis spherical $\mathrm{TiO}_{2}$ and its photo-degradation property on salicylic acid," Applied Surface Science, vol. 258, no. 18, pp. 6891-6896, 2012.

[13] Y. Zhu and S. Kaskel, "Comparison of the in vitro bioactivity and drug release property of mesoporous bioactive glasses (MBGs) and bioactive glasses (BGs) scaffolds," Microporous and Mesoporous Materials, vol. 118, no. 1-3, pp. 176-182, 2009.

[14] H. S. Wang, X. H. Gao, Y. A. Wang et al., "Bio-templated synthesis of mesoporous bioactive glass with a hierarchical pore structure," Materials Letters, vol. 76, pp. 237-239, 2012.

[15] T. Zhang, X. Dong, and Z. T. Zhang, "The modification and super adiabatic performance of channel type of microporous and mesoporous molecular sieve," Journal of High School Chemistry, vol. 35, pp. 689-694, 2014.

[16] X. Y. Fu, J. F. Huang, L. Y. Cao et al., "Photocatalytic performance of different morphology of hydrogen lanthanum oxide nanocrystals," Journal of Silicate, vol. 48, pp. 1158-1166, 2013.

[17] G. C. Liu, Z. Jin, X. B. Zhang et al., "La doping BiVO4 micro ball of hydrothermal synthesis and light catalytic properties," Journal of Chinese Nonferrous Metals, vol. 23, pp. 793-801, 2013.

[18] H. Deng, J. Lu, G. Li, G. Zhang, and X. Wang, "Adsorption of methylene blue on adsorbent materials produced from cotton stalk," Chemical Engineering Journal, vol. 172, no. 1, pp. 326-334, 2011.

[19] R.-L. Liu, Y. Liu, X.-Y. Zhou, Z.-Q. Zhang, J. Zhang, and F.-Q. Dang, "Biomass-derived highly porous functional carbon fabricated by using a free-standing template for efficient removal of methylene blue," Bioresource Technology, vol. 154, pp. 138-147, 2014. 

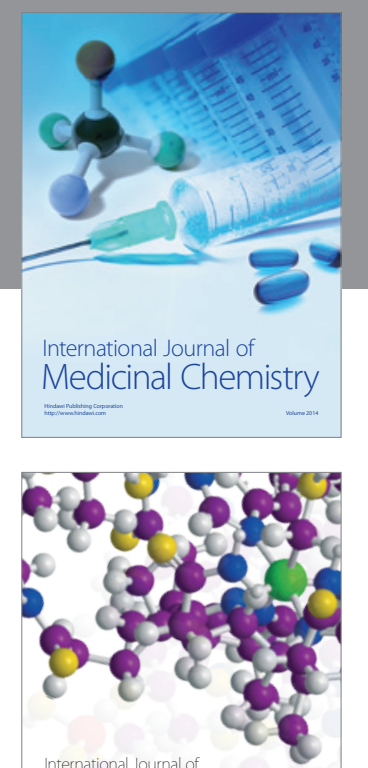

\section{Carbohydrate} Chemistry

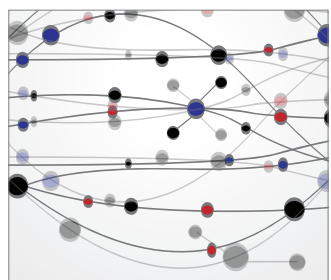

The Scientific World Journal
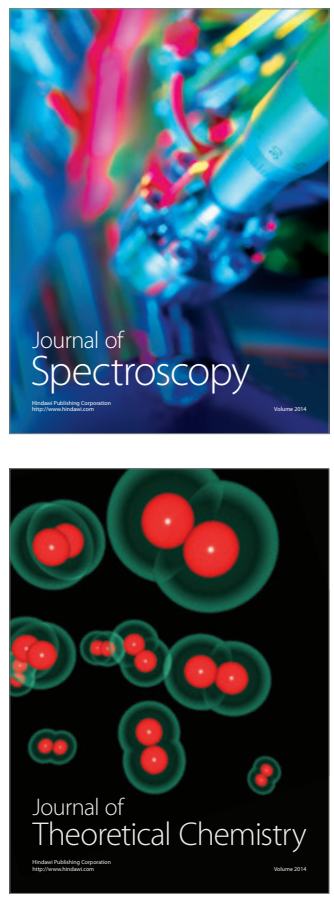
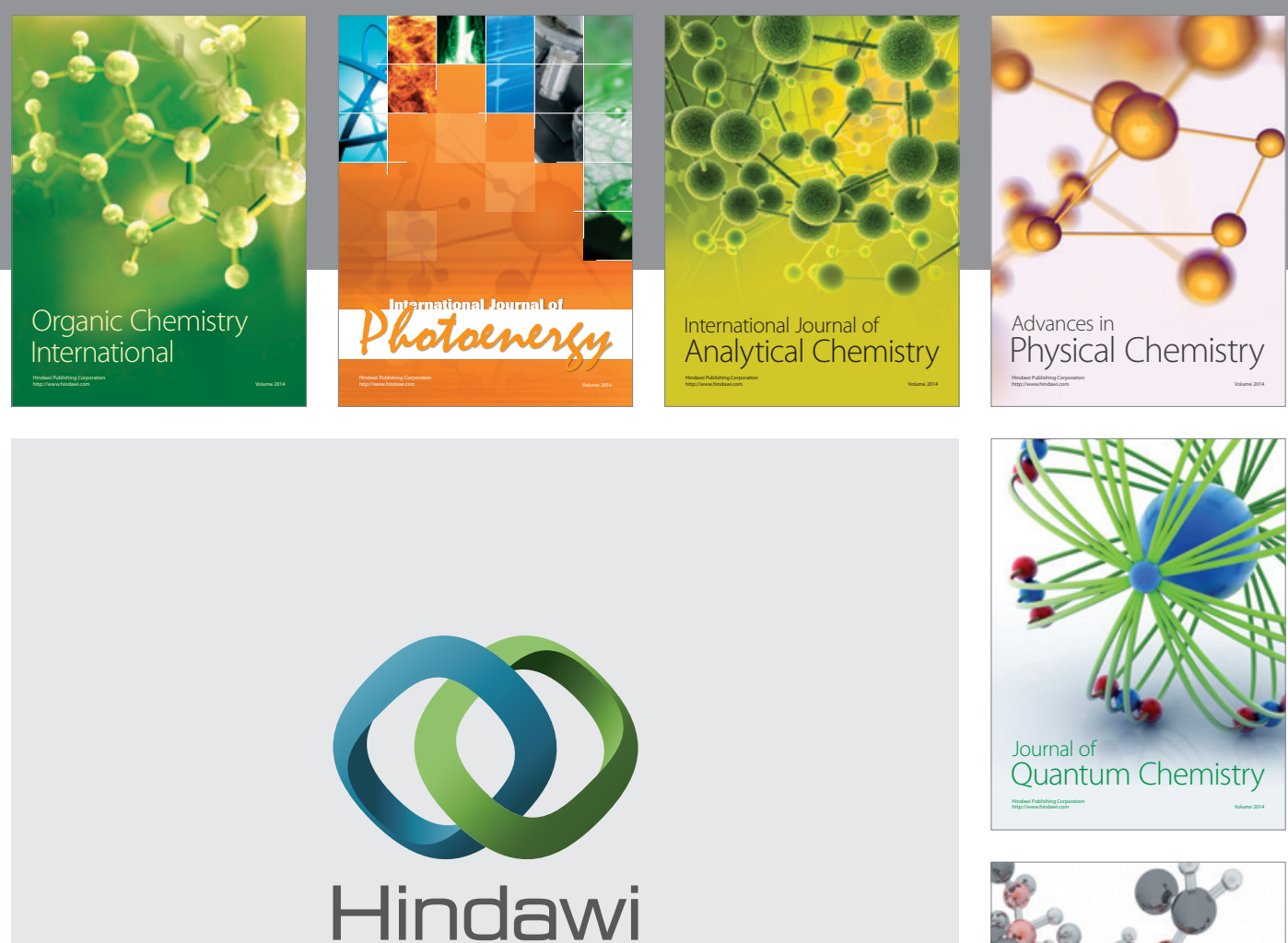

Submit your manuscripts at

http://www.hindawi.com

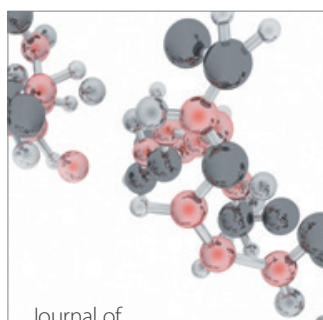

Analytical Methods

in Chemistry

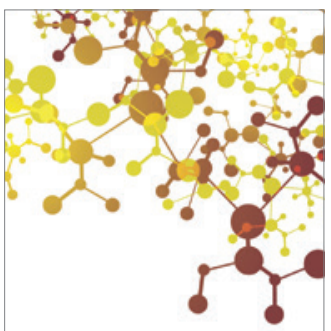

Journal of

Applied Chemistry

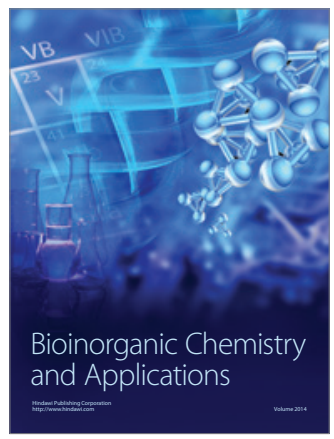

Inorganic Chemistry
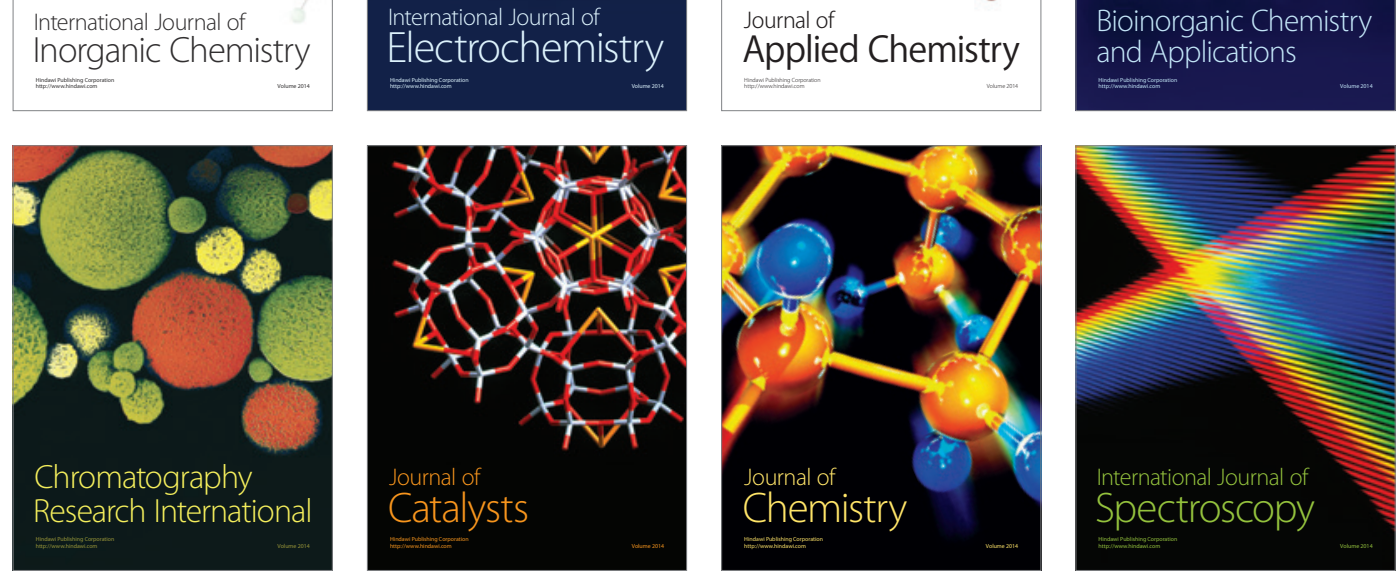